\title{
Baseline Brain Metabolism in Resistant Depression and Response to Transcranial Magnetic Stimulation
}

\author{
Marie-Laure Paillère Martinot*, ${ }^{*, 2}$, Jean-Luc Martinot ${ }^{2,3}$, Damien Ringuenet ${ }^{4}$, André Galinowski ${ }^{5}$, \\ Thierry Gallarda ${ }^{5}$, Frank Bellivier ${ }^{6}$, Jean-Pascal Lefaucheur ${ }^{7}$, Hervé Lemaitre ${ }^{2,3}$ and Eric Artiges ${ }^{2,3,8}$ \\ 'AP-HP, Department of Adolescent Psychopathology and Medicine, Maison de Solenn, Cochin Hospital, University Paris Descartes, Sorbonne \\ Paris Cité, Paris, France; 'INSERM, U 1000, Research Unit 'Imaging \& Psychiatry', University Paris-Sud and University Paris Descartes, \\ Orsay, France; ${ }^{3}$ CEA, DSV, I²BM, Service Hospitalier Frédéric Joliot, Orsay, France; ${ }^{4}$ AP-HP, Department of Psychiatry and Addictology, \\ Paul Brousse Hospital, University Paris-Sud, Villejuif, France; ${ }^{5} \mathrm{SHU}$ Sainte-Anne Hospital and INSERM, U 894, Psychiatry and Neuroscience \\ Center, University Paris Descartes, Paris, France; ' ${ }^{2}$ P -HP, Department of Psychiatry, and INSERM, U 955, IMRB, Psychiatry Genetics, \\ Henri Mondor-Albert Chenevier Hospital, Créteil, France; ${ }^{7}$ AP-HP, Physiology Department, Henri Mondor-Albert Chenevier Hospital, \\ University Paris 12, Créteil, France; ${ }^{8}$ Psychiatry Department 9 I Gl6, Orsay Hospital, Orsay, France
}

Neuroimaging studies of patients with treatment-resistant depression (TRD) have reported abnormalities in the frontal and temporal regions. We sought to determine whether metabolism in these regions might be related to response to repetitive transcranial magnetic stimulation (TMS) in patients with TRD. Magnetic resonance images and baseline resting-state cerebral glucose uptake index (gluMI) obtained using ${ }^{18}$ F-fluorodeoxyglucose positron emission tomography were analyzed in TRD patients who had participated in a doubleblind, randomized, sham-controlled trial of prefrontal $10 \mathrm{~Hz}$ TMS. Among the patients randomized to active TMS, 17 responders, defined as having 50\% depression score decrease, and 14 nonresponders were investigated for prestimulation glucose metabolism and compared with 39 healthy subjects using a voxel-based analysis. In nonresponders relative to responders, gluMl was lower in left lateral orbitofrontal cortex (OFC), and higher in left amygdala and uncinate fasciculus. OFC and amygdala gluMl negatively correlated in nonresponders, positively correlated in responders, and did not correlate in healthy subjects. Relative to healthy subjects, both responders and nonresponders displayed lower gluMl in right dorsolateral prefrontal (DLPFC), right anterior cingulate (ACC), and left ventrolateral prefrontal cortices. Additionally, nonresponders had lower gluMl in left DLPFC, ACC, left and right insula, and higher gluMl in left amygdala and uncus. Hypometabolisms were partly explained by gray matter reductions, whereas hypermetabolisms were unrelated to structural changes. The findings suggest that different patterns of frontal-temporal-limbic abnormalities may distinguish responders and nonresponders to prefrontal magnetic stimulation. Both preserved OFC volume and amygdala metabolism might precondition response to TMS.

Neuropsychopharmacology (201 I) 36, 2710-2719; doi:10.1038/npp.2011.161; published online 17 August 201 I

Keywords: depression; fluorodeoxyglucose; magnetic resonance imaging; positron emission tomography; transcranial magnetic stimulation; treatment response

\section{INTRODUCTION}

Treatment-resistant depression (TRD) occurs in 20 to $30 \%$ of depressed patients and leads to severe disability (Greden, 2001). Neuroimaging studies focused on TRD patients are rare, and have reported decreased blood flow or glucose metabolism in prefrontal regions such as the anterior cingulate cortex (ACC) (Mayberg et al, 1994, 1997b; Drevets et al, 1997; Ketter et al, 2001; Konarski et al, 2007), and in

*Correspondence: Dr M-L Paillère Martinot, AP-HP, Department of Adolescent Psychopathology and Medicine, Maison de Solenn, Hôpital Cochin, 97 Boulevard de Port-Royal, 75014 Paris, France, Tel: + 33 I5 84I 2426, Fax: + $331584 \mid$ 2808, E-mail: ml.paillere@cch.aphp.fr Received 9 March 20 I ; revised I July 20 II; accepted I 5 July 201 I anterior temporal cortices (Mayberg et al, 1994). Differential frontal-temporal changes have been reported in medication responder and nonresponder subgroups of depressed patients. Responders to antidepressants were found to have lower pretreatment glucose metabolism in both left amygdala region and temporal cortex, and in bilateral frontal cortices (Little et al, 2005). In line with these findings, higher resting-state hippocampus-amygdala blood flow was found in a group of TRD compared with nonresistant patients and healthy controls (Hornig et al, 1997). Cortical-limbic balance involving orbitofrontal cortex (OFC), ACC, hippocampus, and dorsolateral prefrontal cortex (DLPFC) might differentiate responders from nonresponders to antidepressant medications (Seminowicz et al, 2004). 
Repetitive transcranial magnetic stimulation (TMS) has been proposed as a potential treatment for TRD (George et al, 1995; Pascual-Leone et al, 1996) that can easily be applied in clinical settings and is well tolerated. This procedure aims at stimulating the left DLPFC in order to increase brain metabolism in prefrontal areas using high stimulation frequencies $(>5 \mathrm{~Hz})$. However, although a number of studies have now demonstrated the clinical effectiveness of TMS in TRD patients (Lam et al, 2008), the effects remain variable and factors predictive of response remain poorly understood (Loo and Mitchell, 2005; Herrmann and Ebmeier, 2006; Lisanby et al, 2009). As this therapy is time consuming and costly, it is necessary to determine whether brain factors could influence or predict outcome. High-frequency TMS of prefrontal regions, as used to treat depression, has been shown to modulate ACC and caudate nucleus activity in healthy subjects (Barrett et al, 2004). In addition, blood flow decreases in OFC and ACC have been reported after treatment with highfrequency TMS (Nadeau et al, 2002) in responders to TMS. So far, however, it is not clear whether prestimulation functional anatomy could influence TMS efficacy and predict response.

We a priori hypothesized that TRD patients who were nonresponders to TMS would have specific alterations in those frontal-temporal-limbic regions involved in TRD and in TMS effects, and also in the uncinate fasciculus, a frontal-temporal fiber tract that we have reported to be altered in patients with affective disorders (Houenou et al, 2007). We used $\left[{ }^{18} \mathrm{~F}\right]$-fluorodeoxyglucose positron emission tomography $\left(\left[{ }^{18} \mathrm{~F}\right]\right.$-FDG-PET) to determine whether resting functional brain characteristics at baseline could differentiate responders from nonresponders to $10 \mathrm{~Hz}$ TMS in a group of TRD patients. Additionally, we searched for a potential influence of structural anatomy on metabolism, and for functional correlations within the regions involved in response to TMS.

\section{SUBJECTS AND METHODS}

The investigation was performed in accordance with the Declaration of Helsinki. The study was approved by the ethics committee Ile-de-France 6, Paris. Written informed consent was obtained from all subjects after full description of the study.

\section{Participants}

Pretrial PET and magnetic resonance imaging (MRI) data from the 34 patients who were initially randomized to an active arm in a double-blind, sham-controlled trial of $10 \mathrm{~Hz}$ TMS including 48 patients (Paillère Martinot et al, 2009) were considered for the present imaging study. The patients had a DSM-IV-TR diagnosis of Major Depressive Disorder established by clinical interview using the Mini-International Neuropsychiatric Interview (MINI) (Sheehan et al, 1998), with criteria for treatment resistance to at least two trials of antidepressants of different classes given at adequate doses (>150 mg/day in an equivalent dose of imipramine) and duration (at least 4 weeks for each drug).
Exclusion criteria included age $>65$ years, pregnancy, alcohol or substance dependence in the past 6 months, electroconvulsive therapy (ECT) treatment in the past 6 months, any present medical condition, history of epileptic seizures, history of neurological disorders or substantial brain damage, and contraindication to magnetic fields. On examination of the patient medical charts, three patients were excluded; past early-onset alcoholism in 2 patients and a possible diagnosis of fibromyalgia in one patient. Indeed, alcoholism with onset in adolescence might have modified brain structure (Chanraud et al, 2007), and fibromyalgia has been reported to alter brain activity in frontocingulate areas (Burgmer et al, 2009).

Thus, data from 31 patients were analyzed in the present study (mean (SD) age $=47.7$ (7.3); range: 30.5-59.5, 20 women). They had on average 7.4 (4.4) years of education after primary school, and were not paid for their participation.

A total of 39 paid healthy comparison subjects with no personal or family history of psychiatric or neurologic disorder, as assessed by a medical examination, were recruited by word of mouth from community volunteers during the same time period (age: mean $(\mathrm{SD})=45.2$ (11.8); range: 25-62, 25 women; years of education after primary school: mean $(\mathrm{SD})=9.10(4.8)$ ). Patients and healthy subjects did not differ in age $(t=0.99, \mathrm{df}=68, p=0.32)$, gender $\left(\chi^{2}\right.$ test $\left.=0.000, p=0.98\right)$, years of education $(t=1.53, \mathrm{df}=67, p=0.13)$, or Annett's (1970) laterality score (patients: mean $(\mathrm{SD})=85.7(49.3)$, healthy subjects: 89.4 (26.5), $t=0.40, \mathrm{df}=67, p=0.69$ ).

\section{TMS Protocol}

Design. The stimulation procedure and determination of PET-derived targets for TMS have been described elsewhere (Paillère Martinot et al, 2009). Briefly, after scanning, the patients were randomized to treatment with PET-guided, active-standard or sham-standard TMS, and subsequently underwent 10 sessions of $10 \mathrm{~Hz}$ TMS delivered at $90 \%$ motor threshold with 1600 pulses/session, using a Magstim super-rapid device with active and sham air-cooled figureof-eight coils (Magstim, Withland, Dyfed, UK). Guided TMS was on a prefrontal target determined with FDG-PET. Standard stimulation was as usual left prefrontal, $5 \mathrm{~cm}$ anterior to the hot spot of the hand motor cortical region. Patients and raters were blind to TMS modality.

Treatment allocation. The 31 patients in this imaging study had been randomly allocated to standard TMS $(n=9$ in responder group, $n=6$ in nonresponder group) or to PETguided TMS ( $n=8$ in responders, $n=8$ in nonresponders; Table 1). No difference in allocation was found across groups $\left(\chi^{2}=0.31, \mathrm{df}=1, p=0.56\right)$. No patient randomized to sham TMS was analyzed in the present study, as response to sham TMS might be related to different mechanisms than response to active treatment, and as there were only 3 responders out of 14 sham-treated patients in that group.

\section{Clinical Assessment}

Baseline assessment was performed on the day before scanning using the Montgomery-Åsberg Depression Rating 
Table I Demographic, Clinical, and Stimulation Characteristics of Responders and Nonresponders to rTMS

\begin{tabular}{|c|c|c|c|}
\hline Variable & $\begin{array}{l}\text { Responders } \\
(n=17)\end{array}$ & $\begin{array}{l}\text { Nonresponders } \\
\quad(n=14)\end{array}$ & $\begin{array}{c}P- \\
\text { value }^{a}\end{array}$ \\
\hline Gender (M/F), $n$ & $6 / 11$ & $5 / 9$ & 0.98 \\
\hline Age, years, mean (SD) & $48.7(6.2)$ & $46.4(8.6)$ & 0.40 \\
\hline Education, years ${ }^{\mathrm{b}}$, mean (SD) & $7.1(4.6)$ & $7.8(4.3)$ & 0.69 \\
\hline $\begin{array}{l}\text { Annett scale laterality score, } \\
\text { mean (SD) }\end{array}$ & $75.9(64.5)$ & $98.5(3.2)$ & 0.22 \\
\hline Age at onset, years mean (SD) & $30.1(10.9)$ & $26.1(8.2)$ & 0.27 \\
\hline $\begin{array}{l}\text { Duration of illness, years, } \\
\text { mean (SD) }\end{array}$ & $18.2(10.9)$ & $20.1(6.6)$ & 0.58 \\
\hline $\begin{array}{l}\text { Duration of episode, years, } \\
\text { mean (SD) }\end{array}$ & $3.6(2.7)$ & $2.1(1.7)$ & 0.10 \\
\hline Family depression, $n(\%)$ & $9(53)$ & $5(36)$ & 0.34 \\
\hline Bipolar resistant depression, n (\%) & $6(35)$ & $5(36)$ & 0.98 \\
\hline $\begin{array}{l}\text { Number of depressive episodes, } \\
\text { mean (SD) }\end{array}$ & $3.3(1.6)$ & $4.07(2.09)$ & 0.30 \\
\hline $\begin{array}{l}\text { Number of manic episodes, } \\
\text { mean (SD) }\end{array}$ & $0.6(1.2)$ & $0.4(0.8)$ & 0.44 \\
\hline $\begin{array}{l}\text { Comorbid Anxiety Disorder, } \\
\text { n (\%) }\end{array}$ & $3(18)$ & $4(29)$ & 0.47 \\
\hline $\begin{array}{l}\text { Tyrer anxiety scale score, } \\
\text { mean (SD) }\end{array}$ & $20.2(1.8)$ & $20.7(2.1)$ & 0.87 \\
\hline $\begin{array}{l}\text { Left-guided/right-guided/left } \\
\text { standard rTMS, n }\end{array}$ & $6 / 2 / 9$ & $3 / 5 / 6$ & 0.26 \\
\hline I8-FDG dose, Mbq, mean (SD) & |54.| (7.6) & I57.3 (I0.8) & 0.36 \\
\hline \multicolumn{4}{|l|}{ MADRS scores, mean (SD) } \\
\hline Baseline & $32.1(7.7)$ & $35.1(6.3)$ & 0.26 \\
\hline End point & $8.9(4.0)$ & $27.6(9.0)$ & $<0.001$ \\
\hline \% Improvement & $71.0(12.8)$ & $21.5(20.2)$ & $<0.001$ \\
\hline \multicolumn{4}{|l|}{ HDRS scores, mean (SD) } \\
\hline Baseline & $25.2(4.5)$ & $26.7(5.7)$ & 0.41 \\
\hline End point & $9.0(3.3)$ & $21.6(6.8)$ & $<0.001$ \\
\hline$\%$ Improvement & $63.7(12.2)$ & $19.0(16.1)$ & $<0.001$ \\
\hline \multicolumn{4}{|l|}{ Ongoing medications, n (\%) } \\
\hline Antidepressants & $8(47)$ & $6(43)$ & 0.81 \\
\hline Mood stabilizers & $5(29)$ & $6(43)$ & 0.48 \\
\hline Antipsychotics & $7(4 \mid)$ & $7(50)$ & 0.37 \\
\hline Benzodiazepines & $7(4 I)$ & $8(57)$ & 0.28 \\
\hline \multicolumn{4}{|l|}{ Past medications, n (\%) } \\
\hline Antidepressants & $17(100)$ & $14(100)$ & $>0.99$ \\
\hline Mood stabilizers & $9(53)$ & $9(64)$ & 0.72 \\
\hline Antipsychotics & $8(47)$ & $10(7 \mid)$ & 0.27 \\
\hline Benzodiazepines & $11(65)$ & II (78) & 0.46 \\
\hline ECT & $6(35)$ & $7(50)$ & 0.92 \\
\hline
\end{tabular}

Abbreviations: ECT, electroconvulsive therapy; 18-FDG, 18-fluorodeoxyglucose; HDRS, Hamilton depression rating scale, 21 items; MADRS, Montgomery \& Äsberg Depression Rating Scale; rTMS, repetitive transcranial magnetic stimulation.

aThe $\chi^{2}$ tests, Fisher's exact tests, and $t$-tests were used when appropriate. All statistics were two tailed.

${ }^{\text {b }}$ Years of education after primary school.

Scale (MADRS) (Montgomery and Asberg, 1979), the 21item Hamilton Depression Rating Scale (Ham-D) (Hamilton, 1960), and the Tyrer anxiety scale (Tyrer et al, 1984).
Response to TMS, defined as at least $50 \%$ decrease from baseline MADRS score, as in another TMS study of depressed patients (O'Reardon et al, 2007), was assessed using the last clinical assessment performed just after the final TMS session. Among the actively treated patients, 17 were responders and 14 were nonresponders to TMS.

\section{Treatments}

Previous medication was titrated down to a minimal dose that did not lead to significant clinical worsening for at least 2 weeks before scanning. In all, 5 responders and 4 nonresponders had a stage II resistance (failure to at least two adequate trials of antidepressants) according to Thase and Rush criteria (Thase and Rush, 1997), 9 responders and 5 nonresponders a stage III (stage II and failure to tricyclics), 2 responders and 5 nonresponders a stage IV (stage III and failure to monoamine oxidase inhibitors (MAOIs)), and one patient had a stage $\mathrm{V}$ resistance (stage IV and ECT resistance) in the responder to TMS group (Supplementary Table S1).

\section{Comorbidities}

A total of 7 patients met criteria for a comorbid diagnosis of anxiety disorder (panic disorder with or without agoraphobia, or generalized anxiety disorder); 11 patients had resistant depression in the course of bipolar disorder. Among the latter patients, three had a bipolar II type disorder, all of which were in the nonresponder group. These comorbid diagnoses were equally distributed in both groups (Table 1).

\section{Scanning Protocols}

All participants were investigated at rest. They were instructed to lie and relax in the PET camera with eyes closed, in a quiet room with low dimmed light. Head movement was restricted with an individually molded thermoplastic mask.

$\left[{ }^{18} \mathrm{~F}\right]$-FDG-PET 3D images were obtained following a transmission scan for attenuation correction from a Siemens ECAT EXACT HR + tomograph that collects 63 simultaneous slices (intrinsic in-plane resolution: $4.3 \mathrm{~mm}$; voxel size: $2.42 \times 2.42 \times 2.43 \mathrm{~mm}^{3}$ ). A summed image corresponding to the attenuation- and decay-corrected uptake of the $\left[{ }^{18} \mathrm{~F}\right]-\mathrm{FDG}$, expressed in activity concentration $(\mathrm{Bq} / \mathrm{ml})$, was obtained from two 3D time frames $(10 \mathrm{~min}$ each) collected 30-50 min after intravenous injection of the radioligand. The mean (SD) injected ${ }^{18} \mathrm{~F}$-fluorodeoxyglucose radioactivity was $155.6(9.25) \mathrm{MBq}$ in the patients (see Table 1 for doses in groups), and 155.78 (19.65) MBq in healthy subjects $(t=0.04, \mathrm{df}=67, p=0.96)$.

The 3D structural MRIs were acquired on a 1.5 Tesla GE Signa scanner (General Electrics Medical Systems, Milwaukee, WI) using a T1-weighted spoiled gradientrecalled sequence (124 contiguous slices; field of view $24 \mathrm{~cm}$; $256 \times 256$ matrix; voxel size: $\left.0.94 \times 0.94 \times 1.3 \mathrm{~mm}^{3}\right)$.

Each of the $\left[{ }^{18} \mathrm{~F}\right]$-FDG-PET and T1-weighted images were visually inspected for artifacts. 


\section{Image Processing}

All of the $\left[{ }^{18} \mathrm{~F}\right]$-FDG-PET and structural images were processed with Statistical Parametric Mapping software package (SPM5, Wellcome Department of Cognitive Neurology, University College, London, UK; http://www.fil.ion. ucl.ac.uk/spm), implemented on Matlab (The Mathworks, Natik, MA; http://www.mathworks.com).

Structural preprocessing. To take into account a possible effect of structure on $\left[{ }^{18} \mathrm{~F}\right]-\mathrm{FDG}$ images, voxel-based morphometry (VBM) was performed using the unified segmentation implemented in SPM5 (Ashburner and Friston, 2005). T1-weighted images were spatially normalized, segmented into gray and white matter, and modulated before being smoothed with a $10-\mathrm{mm}$ full-width halfmaximum (FWHM) isotropic Gaussian kernel.

$\left[{ }^{18} F\right]-F D G-P E T$ preprocessing. For the healthy subject group, each $\left[{ }^{18} \mathrm{~F}\right]-\mathrm{FDG}$ image was coregistered to its corresponding $\mathrm{T} 1$-weighted image using a rigid-body model. Transformation matrices obtained during the spatial normalization of the T1-weighted images to the MNI template were then applied to the resulting $\left[{ }^{18} \mathrm{~F}\right]-\mathrm{FDG}$ images. The mean image of these $\left[{ }^{18} \mathrm{~F}\right]-F D G$ images was computed, providing a $\left[{ }^{18} \mathrm{~F}\right]-\mathrm{FDG}$ template that was then smoothed with an 8-mm FWHM isotropic Gaussian kernel. Finally, the $\left[{ }^{18} \mathrm{~F}\right]-\mathrm{FDG}$ images of all subjects were spatially normalized to this template and smoothed with an 8-mm FWHM isotropic Gaussian kernel.

\section{Statistical Analysis}

Demographic and clinical data. Analyses were performed using JMP 8 software from SAS (SAS Institute, SAS Campus Drive, Cary, NC). Unpaired $t$-tests and $\chi^{2}$ tests were used for comparing continuous and categorical variables, respectively, in between-group comparisons. Statistical significance was defined as $p<0.05$, two tailed.

Image analysis. Voxel-wise comparisons were performed using SPM5 within the framework of the general linear model (GLM). All results were obtained within the mask of the hypothesized regions of interest, drawn using Wake Forest University (WFU) PickAtlas (Maldjian et al, 2003), including inferior frontal, middle frontal, superior frontal, medial frontal, and orbital gyri, ACC, inferior temporal and parahippocampal gyri, insula, amygdala, caudate, and 'extra-nuclear' fiber tracts. Brain locations were reported as $x, y$, and $z$ coordinates in Montreal Neurologic Institute (MNI) space and WFU PickAtlas was used to convert MNI coordinates into Talairach coordinates. The Talairach and Tournoux atlas (Talairach and Tournoux, 1988) was used to identify brain regions and approximate Brodmann areas (BA).

MRI image analysis. Group comparisons of structural images were performed on gray matter and white matter tissues using an analysis of covariance (ANCOVA) with group (nonresponders, responders, and healthy subjects) as between-subject factor and age, gender, and total intracranial volume as confounding covariates. An F-test was used to compare the three groups. Significance threshold was set at $p<0.05$, false discovery rate (FDR) corrected for multiple comparisons.

PET image analysis. The $\left[{ }^{18} \mathrm{~F}\right]-\mathrm{FDG} 3 \mathrm{D}$ images were entered into the GLM within SPM5; in order to control for global glucose uptake effects, we used proportional scaling global normalization, yielding an index of regionalrelative to global- glucose uptake (gluMI). Baseline gluMI values were compared between responder, nonresponder patients, and healthy subjects using an ANCOVA, with group as between-subject factor and age and gender as confounding covariates. An F-test was used to compare the three groups. Significance threshold was set at $p<0.05$, FDR corrected for multiple comparisons. Thereafter, post hoc $t$-tests were performed within the regions where the F-test was significant. The 'healthy group versus responders', 'healthy group versus nonresponders', and the 'responders versus nonresponders' contrasts were examined. Significance was set at $p<0.001$, uncorrected. Cluster significance thresholds (extent threshold) were set at 10 contiguous voxels (voxel size $=8 \mathrm{~mm}^{3}$ ) to reduce type I errors introduced by potential noise.

PET-MRI analysis. Relations between gluMI and structure were assessed using the biological parametric mapping (BPM) toolbox (Casanova et al, 2007). BPM combines information from different imaging modalities on a voxelwise basis using the GLM. Imaging variables are integrated on a voxel-wise basis, and hence each voxel has a unique regression design that includes the value of each imaging modality for that voxel. Group comparisons of regional cerebral gluMI after accounting for volumetric changes were performed using an ANCOVA with group as betweensubject factor, age and gender as basic confounding covariates, and structural images as voxel-dependent confounding covariate. The $t$-tests were performed within the same regions where the F-test of the PET analysis was significant. The 'healthy group versus responders', 'healthy group versus nonresponders', and the 'responders versus nonresponders' contrasts were examined. Significance was set at $p<0.001$, uncorrected. Cluster significance thresholds (extent threshold) were set at 10 contiguous voxels (voxel size $=8 \mathrm{~mm}^{3}$ ).

Correlation analyses. In order to explore the relationships between gluMI in different regions (PET regional correlations) or between gluMI and clinical scores (PET-clinical symptoms correlations), individual gluMI values at significant peak voxels were extracted from the SPM F-map. Correlation analyses were performed with JMP 8. All correlations were examined using ANCOVAs with group as between-subject factor.

As our aim was to examine intergroup differences, we report herein the interaction effects between groups and correlations. Significance was set at $p<0.05$ Bonferroni corrected, for all ANCOVAs. The post hoc correlations were performed in each group using Pearson's $r$ test, when an interaction effect was significant. 


\section{RESULTS}

\section{Demographic, Clinical, and Treatment Characteristics of the Patients}

Responders to TMS did not differ from nonresponders with regard to clinical symptoms at baseline, disease history, comorbidities, family history of depression, TMS parameters, and type of ongoing and past treatments including antidepressants, mood stabilizers, antipsychotics, benzodiazepines, or ECT prescriptions. No difference was found between subgroups regarding the resistance stage $\left(\chi^{2}=1.48, \mathrm{df}=2, p=0.48\right.$; Table 1 and Supplementary Table S1).

Additionally, bipolar (BP) and unipolar (UP) TRD patients did not differ regarding age (BP: mean age $(S D)=47$ (5.9), UP: mean age (SD) $=47.8$ (8.1), Student's $t$-test score $=0.27, \mathrm{df}=29, p=0.79$ ), gender (BP: 6 women, 5 men, UP: 14 women, 6 men, $\left.\chi^{2}=0.73, \mathrm{df}=1, p=0.39\right)$, age at onset (BP: 26.3 (7.7), UP: $29.3(10.8), t$-score $=0.82$, $\mathrm{df}=28, p=0.42)$, duration of illness (BP: 20.7 (7.3) years, UP: $18.2(10.0)$ years, $t$-score $=0.73, \mathrm{df}=28, p=0.47)$, duration of depressive episode (BP: 3.4 (3.3) years, UP: 2.7(1.8) years, $t$-score $=0.77, \mathrm{df}=28, p=0.44)$, number of depressive episodes (BP: 4.2 (1.9), UP: 3.4 (1.8), $t$ score $=1.07, \mathrm{df}=27, \quad p=0.29$ ), or family history (BP: yes $=4$, UP: yes $=10, \chi^{2}=0.54, \mathrm{df}=1, p=0.46$ ). UP and $\mathrm{BP}$ did not differ in depression scores improvement rate (BP: $55.6 \% \quad(9.0 \%), \quad \mathrm{UP}: 44.8 \% \quad(6.7 \%), t$-score $=0.96$, $\mathrm{df}=29, p=0.34)$.

\section{PET Findings}

There was a main effect of group on the $\left[{ }^{18} \mathrm{~F}\right]$-FDG-PET images before accounting for structural differences, as evidenced by the results of the F-test, which revealed a pattern of several regions (Table 2). The post hoc $t$-tests performed in those regions showed that both responders and nonresponders to TMS had, in comparison with healthy subjects, lower prefrontal gluMI in right ACC, right DLPFC, and left ventrolateral prefrontal cortex (VLPFC). Additionally, nonresponders had lower gluMI in left ACC, left DLPFC, right and left anterior insula, and left OFC. They also displayed higher gluMI in left inferior temporal-limbic regions including uncus, left amygdala, and left uncinate fasciculus. No significantly higher gluMI values were found in responders in comparison with healthy subjects. In nonresponders relative to responders, gluMI was higher in the left amygdala, uncinate fasciculus, and anterior commissure, and lower in the left OFC (Table 2 and Figure 1).

\section{MRI Corrected PET Findings}

When accounting for volume differences, regions that survived the volume correction included right ACC, right and left insula, left DLPFC, left uncus at BA20, and left amygdala (Table 2). Volume fully accounted for lower gluMI in the left OFC, left VLPFC (BA9/47), left ACC, and

Table 2 Differences in Regional Cerebral Glucose Metabolism Index between 17 Responders and I4 Nonresponders to rTMS Compared with 39 Healthy Subjects

\begin{tabular}{|c|c|c|c|c|c|c|c|c|c|c|c|c|c|c|c|}
\hline \multirow{3}{*}{$\begin{array}{l}\text { Brain region } \\
\text { Right MFG }\end{array}$} & \multicolumn{6}{|c|}{ Main effect of group ${ }^{a}$} & \multicolumn{5}{|c|}{ Group comparison, $t$-value ${ }^{b}$} & \multicolumn{4}{|c|}{ Volume corr. ${ }^{c}, t$-value ${ }^{b}$} \\
\hline & \multirow{2}{*}{$\begin{array}{c}\text { BA } \\
9\end{array}$} & \multirow{2}{*}{$\begin{array}{c}\begin{array}{c}\text { Cluster } \\
\text { size }^{\mathbf{d}, \mathbf{e}}\end{array} \\
317\end{array}$} & \multicolumn{3}{|c|}{$\begin{array}{c}x, y, z \\
\text { MNI coordinates }{ }^{f}\end{array}$} & \multirow{2}{*}{$\begin{array}{c}\text { F-test }^{\mathbf{a}} \\
11.42\end{array}$} & \multirow{2}{*}{$\begin{array}{c}\mathbf{R}<\mathbf{H S} \\
3.55\end{array}$} & \multirow{2}{*}{$\begin{array}{c}\mathbf{N R}<\mathbf{H S} \\
4.14\end{array}$} & \multirow{2}{*}{$\begin{array}{c}\mathbf{N R}>\mathbf{H S} \\
-\end{array}$} & \multirow{2}{*}{$\begin{array}{c}\mathbf{R}>\mathbf{N R} \\
--\end{array}$} & \multirow{2}{*}{$\begin{array}{c}\mathbf{R}<\mathbf{N R} \\
--\end{array}$} & \multirow{2}{*}{$\begin{array}{c}\mathbf{R}<\mathbf{H S} \\
--\end{array}$} & \multirow{2}{*}{$\begin{array}{c}\mathbf{N R}<\mathbf{H S} \\
-\end{array}$} & \multirow{2}{*}{$\begin{array}{c}\mathbf{N R}>\mathbf{H S} \\
--\end{array}$} & \multirow{2}{*}{$\begin{array}{r}\mathbf{R}<\mathbf{N R} \\
--\end{array}$} \\
\hline & & & 34 & 12 & 38 & & & & & & & & & & \\
\hline Right insula & 45 & 191 & 36 & 22 & 12 & 9.75 & - & 3.97 & - & - & - & - & 3.25 & - & - \\
\hline Right ACC & 32 & 850 & 12 & 34 & 22 & 13.67 & 3.37 & 4.85 & - & - & - & 3.56 & 4.22 & - & - \\
\hline Left ACC & 32 & - & -2 & 38 & 28 & 9.03 & - & 4.07 & - & - & - & - & - & - & - \\
\hline Left MFG & 9 & 26 & -40 & 28 & 42 & 7.86 & - & 3.93 & - & - & - & - & 3.53 & - & - \\
\hline Left IFG & 9 & 630 & -40 & 8 & 38 & 9.86 & 3.49 & 3.84 & - & - & - & 3.32 & - & - & - \\
\hline Left IFG & 47 & - & -48 & 14 & 24 & 9.60 & 4.10 & 3.46 & - & - & - & 3.57 & - & - & - \\
\hline Left MFG & 46 & - & -44 & 26 & 26 & 9.52 & - & 4.18 & - & - & - & 3.32 & 3.43 & - & - \\
\hline Left MFG (orbital) & 10 & 43 & -32 & 50 & -4 & 8.17 & - & 3.94 & - & 3.79 & - & - & - & - & - \\
\hline Left insula & 45 & 60 & -32 & 26 & 8 & 8.28 & - & 3.80 & - & - & - & 3.77 & 3.22 & - & - \\
\hline Left amygdala & - & 439 & -24 & -2 & -28 & 13.10 & - & - & 4.84 & - & 4.60 & - & - & 4.79 & 4.57 \\
\hline Left ITG (uncus) & 20 & - & -32 & -10 & -38 & 8.82 & - & - & 4.20 & - & - & - & - & 4.33 & - \\
\hline Left UF & - & 93 & -32 & 4 & -10 & 8.41 & - & - & 3.60 & - & 3.87 & - & - & 3.51 & 3.80 \\
\hline Left AC & - & - & -32 & -8 & -10 & 9.05 & - & - & - & - & 4.23 & - & - & - & 4.08 \\
\hline
\end{tabular}

Abbreviations: AC, anterior commissure; ACC, anterior cingulate cortex; BA, Brodmann area; ellipses, no cluster retrieved by comparison, or not applicable;

HS, healthy subject; IFG, inferior frontal gyrus; ITG, inferior temporal gyrus; MFG, middle frontal gyrus; NR, nonresponder; R, responder; UF, uncinate fasciculus.

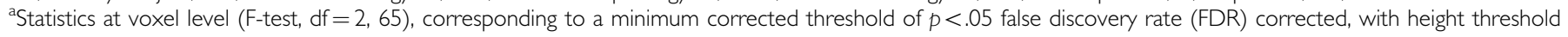
$\mathrm{F}=6.97$, and extent threshold $k=10$ voxels.

${ }^{\mathrm{b}}$ The post hoc test, height threshold was set at $p<0.00 \mathrm{I}$, uncorrected, and extent threshold was set at 10 voxels.

'Volume corr., volume corrected analysis of PET images with TI anatomic images as voxel-dependent confounding covariate.

dEmpty cells indicate that the region is included in the same cluster as the region immediately above.

${ }^{e}$ Cluster size is expressed in number of voxels, with voxel size $=8 \mathrm{~mm}^{3}$.

fMontreal Neurological Institute, in millimeters, for significant peak voxels. 

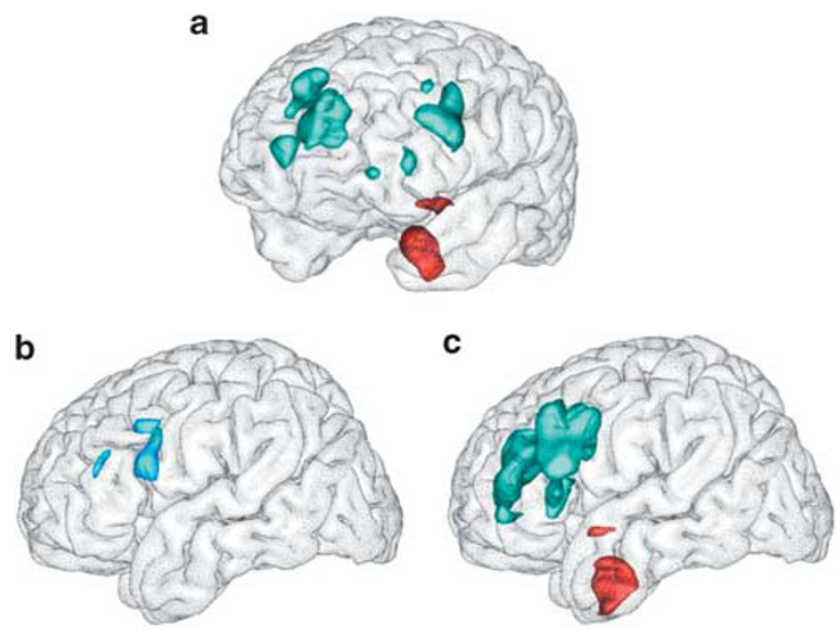

Figure I (Top, left frontal view) Main comparison (F-test) between responders, nonresponders, and healthy subjects. Height threshold: $p<0.05$, false discovery rate corrected; extent threshold: 10 voxels (a). (Bottom, lateral views) The t-maps of baseline regional cerebral glucose metabolism index (gluMl) in 17 responders to rTMS (b, left) and in 14 nonresponders (c, right) compared with 39 healthy subjects, respectively (height threshold: $p<0.00$ I; extent threshold: 10 voxels); red color indicates higher gluMl, and blue indicates lower gluMl. Statistical maps are projected on a normalized brain mesh.

right DLPFC. Higher gluMI was not related to white matter volume in uncinate fasciculus or in anterior commissure.

\section{MRI Findings}

Voxel-based comparison of the GM maps revealed no main effect of group at the $p<0.05$, FDR corrected for multiple comparisons. However, at a more permissive threshold, GM volume reductions were observed in some regions where gluMI was low, including the left OFC (BA10, $t=3.52$, $p<.001$ unc, $k=36$ voxels) in nonresponders relative to responders and relative to healthy subjects $(t=2.87$, $p<.002, k=312$ voxels), and in the left ACC (BA 32, $t=3.40, p<.001$ unc, $k=46)$ in nonresponders relative to healthy subjects. No regional difference was found in white matter maps between responders, nonresponders, and healthy subjects.

\section{FDG-PET Regional Correlations}

Responders, nonresponders, and healthy subjects significantly differed in several PET regional correlations.

Interactions (F-tests). Significant interactions (glu$\mathrm{MI} \times$ group) were found between left $\mathrm{OFC}$ and several regions in which metabolic level was independent of structure. Regions included left amygdala $(\mathrm{F}=8.20$, $\mathrm{df}=2)$, left DLPFC at BA9 $(\mathrm{F}=11.79, \mathrm{df}=2)$, left VLPFC at BA9 $(\mathrm{F}=14.34, \mathrm{df}=2)$, right $\mathrm{ACC}(\mathrm{F}=8.18, \mathrm{df}=2)$, and left uncus $(\mathrm{BA} 20)(\mathrm{F}=8.31, \mathrm{df}=2)$.

Post hoc tests. In the nonresponders, left amygdala gluMI was negatively correlated with gluMI in the left OFC, whereas a positive correlation in the responders and no correlation in the healthy subjects was observed (Figure 2

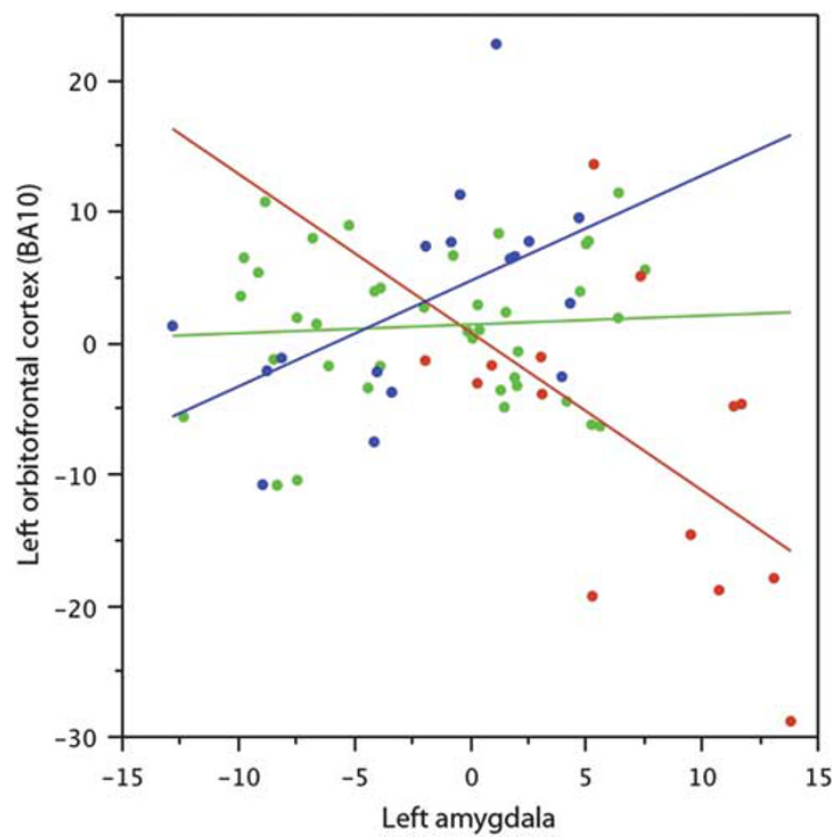

Figure 2 Regional correlations between regional cerebral glucose metabolism index in left frontal BAIO at MNI coordinates $(x=-32$, $y=50, \quad z=-4)$ and in left amygdala $(x=-24, \quad y=-2, \quad z=-28)$. Interaction: $F=8.20, d f=2, p<0.001$. Red indicates negative correlation in nonresponders, Pearson's $r=-0.55, p=0.042$. Blue indicates positive correlation in responders, Pearson's $r=0.52, p=0.031$. Green indicates no correlation in healthy subjects, Pearson's $r=0.07, p=0.68$.

Table 3 Regional Correlations of Glucose Metabolism with Orbitofrontal Cortex across Groups

\begin{tabular}{|c|c|c|c|}
\hline & $\begin{array}{l}\text { Healthy subjects } \\
(n=39)\end{array}$ & $\begin{array}{l}\text { Responders } \\
(n=17)\end{array}$ & $\begin{array}{l}\text { Nonresponders } \\
(n=14)\end{array}$ \\
\hline $\begin{array}{l}\text { Right ACC } \\
\text { (BA32) }\end{array}$ & $r=0.31 ; p=0.06$ & $r=-0.19 ; p=0.47$ & $r=0.77 ; p=0.001$ \\
\hline Left MFG (BA9) & $r=0.19 ; p=0.25$ & $r=-0.20 ; p=0.45$ & $r=0.92 ; p<0.001$ \\
\hline Left IFG (BA9) & $r=0.04 ; p=0.25$ & $r=0.02 ; p=0.45$ & $r=0.92 ; p<0.0001$ \\
\hline $\begin{array}{l}\text { Left uncus } \\
(\text { BA20) }\end{array}$ & $r=0.02 ; p=0.92$ & $r=0.14 ; p=0.58$ & $r=-0.70 ; p=0.005$ \\
\hline Left amygdala & $r=0.07 ; p=0.68$ & $r=0.52 ; p=0.03$ & $r=-0.55 ; p=0.04$ \\
\hline
\end{tabular}

Abbreviations: ACC, anterior cingulate cortex; BA, Brodmann area; IFG, inferior frontal gyrus; MFG, middle frontal gyrus; $p$, probability value; $r$, Pearson's correlation coefficient.

Significant correlations are indicated in bold.

and Table 3). A negative correlation was also found in nonresponders between gluMI in left uncus and left OFC, but no correlation was found in responders or in healthy subjects. Positive correlations were observed in nonresponders, but not in other groups, between OFC and (1) prefrontal regions including left DLPFC at BA9; (2) left VLPFC at BA9; and (3) right ACC.

\section{Clinical Correlations}

No significant interaction (gluMI $\times$ group) was found between gluMI and baseline scores of depression, anxiety 
scores, or between gluMI and change in depression scores after TMS.

\section{DISCUSSION}

Pretreatment brain glucose metabolism at rest and regional volumes were investigated in patients with resistant depression to assess whether these characteristics could differentiate responders and nonresponders to prefrontal TMS. Nonresponders had a pattern of widespread low prefrontal metabolism associated with high temporallimbic resting-state metabolism. Low metabolism was related to variations in GM volume in several regions, notably in left OFC and ACC, where lower GM volume was observed in nonresponders. Uniquely, different correlations between left amygdala and left OFC metabolism distinguished the nonresponders, responders, and healthy subject groups.

\section{Functional Correlates of Response to TMS}

Few studies have examined brain correlates of antidepressant response to high-frequency TMS and most of those studies have used single-photon emission computerized tomography (SPECT) in smaller groups of patients, pooling both medication-resistant and nonresistant depressed patients, with response defined as a 30\% decrease in depression scores (50\% in the present study). Responders to TMS have been found to have higher blood flow in inferior frontal lobe before stimulation (Teneback et al, 1999), or lower blood flow in amygdala (Nadeau et al, 2002) than nonresponders, consistent with our results. Improvement in depression scores with TMS has also been found to positively correlate with blood flow in the anterior cingulate (Langguth et al, 2007) and in the right peri-insular region (Mottaghy et al, 2002).

Consistent with other studies of TRD patients (Videbech, 2000; Konarski et al, 2007), the present findings point to a pattern of decreased prefrontal glucose metabolism, particularly in regions involved in TMS response, including the insula, the ACC, and the OFC. In nonresponders to TMS relative to responders, low glucose metabolism was observed in these regions, and related to lower GM volumes in the left ACC and in the rostral part of the left OFC. In line with this finding, hypometabolism in OFC has been hypothesized to be related to GM reduction in more severely ill patients such as the nonresponders to TMS in our study (Drevets, 1999), whereas normal glucose metabolism in OFC has been reported in medication-resistant depressives with no GM reduction (Drevets, 2000; Drevets et al, 2002). Thus, the present results support that OFC gray matter volume reduction might precondition a negative response to prefrontal TMS.

A functional pattern of prefrontal hypometabolism associated with temporal-limbic hypermetabolism has previously been reported in various groups of depressed patients regardless of resistance to treatment (Konarski et al 2007; Brooks et al, 2009; Savitz and Drevets, 2009). In the present study, a functional amygdala hypermetabolism was only observed in the TRD nonresponders to TMS, regardless of GM volume. Thus, the present findings raise the hypothesis that high metabolic levels of the temporal- limbic regions, particularly the amygdala, might precondition nonresponse to prefrontal TMS.

\section{Frontal-Limbic Correlations in Resistant Depressives}

Distinct correlations were found between OFC and the amygdala in nonresponders, responders, and healthy subjects, indicating different frontal-limbic relations depending on the group. The OFC is involved in coding of the identity of the sensory stimuli and their rewarding properties (Schultz et al, 1998), and is connected with the lateral nuclei of the amygdala, whose activity appears closely related to the context and level of aversiveness of the stimuli (Zald, 2003). Through inhibitory projections to the amygdala, the lateral OFC is consequently involved in controlling information processing, particularly representation of rewards and punishments, and regulates behavior expression and emotional responses (Hooker and Knight, 2006). Thus, GM reductions in this region may disturb interactions between orbital cortex and projections to the amygdala, and to other connected regions such as the cingulate cortex or the striatum. Our findings are consistent with a frontal-limbic dysregulation in the subgroup of nonresponders, leading to decreased frontal cortical regulation of temporal-limbic activation in response to negative stimuli, as has previously been hypothesized in TRD (Mayberg, 1997a). In these patients, and as prefrontal TMS seems to modulate OFC activity (Nadeau et al, 2002), GM reduction of the OFC might partly account for the observed specific resistance to TMS. Such resistance to TMS in these patients may additionally be related to altered white matter tracts in the ventral frontaltemporal-limbic network. Indeed, higher glucose metabolism was detected in left uncinate fasciculus and anterior commissure in nonresponders, independently of white matter volumes. As pointed out by Buchbaum et al (2007), the increased metabolism might reflect increased energy need due to defects in white matter that, in turn, may lead to inefficiency in brain circuitry. The uncinate fasciculus is a bidirectional pathway that links the anterior temporal lobe and amygdala with the medial and orbital prefrontal cortices, and the anterior commissure is a fiber bundle that connects the inferior temporal with sites including the amygdala and OFC (Schmahmann and Pandya, 2006). Thus, it can be speculated that because of changes in the OFC/VLPFC, and connecting white matter tracts such as the uncinate fasciculus, TMS would fail to modulate the prefrontal cortex and temporal-limbic structure activity through the ventral-limbic pathway.

\section{Limitations}

First, although there is no larger FDG-PET study associated with a controlled TMS trial, the short treatment duration might be seen as a limitation. Indeed, TMS has been found to be more effective with longer treatment durations (O'reardon et al, 2007). Thus, some nonresponder patients in this study would perhaps have been responders had they been treated over a longer period of time. However, despite the small number of subjects and short treatment duration, the results of the clinical trial (Paillère Martinot et al, 2009) showed that the chosen protocol accelerated the effect of 
TMS, with a strong effect size (Cohen's $d=0.78$; Cohen, 1988) over sham stimulation, probably accounting for the high response rate in the study.

A second limitation relates to previous medication. It was not possible to withdraw all treatments in these difficult patients, and it was not possible to assess all the dosages prescribed along their illness as they had very long illness durations. However, antidepressant and mood stabilizers were prescribed in usual standard dosages in all the patients. Effects of medication on FDG measures have been studied in a few studies, with inconsistent results. Apparently, brain metabolic responses to antidepressants vary according to the underlying pathophysiology of the patient and the degree of symptomatic improvement (Saxena et al, 2002), and chronic antidepressant drug treatment might reduce metabolism in the amygdala and ventral ACC in depressed subjects showing a positive treatment response only (Drevets et al, 2002). Here, the patients were all similarly resistant to medication according to Thase and Rush criteria, and the number of patients on antidepressants and duration of illness or episode were comparable across groups. Also, the number of patients on antipsychotics and the dosages were very small and similar in both patient groups, as was the number of patients on mood stabilizers; thus, it is unlikely that their effect would have confounded the metabolic findings. Finally, most patients were on low doses of benzodiazepines, which have been reported to induce decreases in glucose metabolic rates in regions such as the basal ganglia, thalamus, or visual cortex (Martinot, 1992). These effects might have blurred some differences with the comparison subjects, but as the prescriptions of patients did not differ across subgroups, it is unlikely that these effects would have confounded the results of direct comparison of responders with nonresponders. Moreover, the metabolic findings are consistent with findings of other studies of untreated depressed patients (Teneback et al, 1999).

Third, in a few studies, resistance to TMS has been associated with previous medication resistance, or anxiety comorbidity (Fregni et al, 2006; Brakemeier et al, 2008; Lisanby et al, 2009). In this study, medication resistance was comparable across responders and nonresponders to TMS. Regarding comorbid anxiety, the number of patients with a comorbid anxiety disorder was similar in both groups, and anxiety scores before scanning were similar across groups, and did not correlate with PET measures, which suggests that nonresponse parameters did not depend on anxiety comorbidity in this group.

Fourth, the pooling of patients with unipolar resistant depression and with resistant depression in the context of history of bipolar disorder may be considered as a limitation. At variance with most previous reports, the present study aimed at assessing $\left[{ }^{18} \mathrm{~F}\right]-\mathrm{FDG}$ in highly treatment-resistant patients notwithstanding the UP/BP dichotomy, rather than to compare UP or BP subgroups. In addition, many imaging studies indicate increased resting-state metabolism in the amygdala in Major Depressive Disorder as well as in BP depression (Savitz and Drevets, 2009). Regarding OFC, resting-state activity has generally been found similarly increased in both UP and BP depressives (Drevets, 2000), although in more severely ill or TRD patients, studies have found no change or decreased function (Mayberg et al, 1994; Savitz \& Drevets, 2009). Thus, putative differences in functional pattern reported in the literature between BP and UP patients are not clearcut.

In addition in the present study, the UP/BP ratios were similar in both responder and nonresponder subgroups, and no difference was found between UP and BP patients regarding improvement rates or depression history, or when comparing UP and BP groups using a similar image analysis, within the same regions of interest and at the same statistical threshold as in the responder/nonresponder analysis. Regarding the responder/nonresponder comparisons, and although the groups were much smaller, the same left amygdala function and left OFC gray matter pattern still distinguished responders from nonresponders within each subgroup. In both the BP and UP subgroups, nonresponders had significantly higher amygdala gluMI (Wilcoxon test, BP: responders, mean (SD) $=-1.36(1.86)$, nonresponders mean $(\mathrm{SD})=9.49(2.04), \chi^{2}=5.63, p=0.02 ; \quad \mathrm{UP}$ : responders, mean $(\mathrm{SD})=-2.19 \quad(1.65)$, nonresponders mean $\left.(\mathrm{SD})=5.21(1.82), \chi^{2}=6.48, p=0.01\right)$. Similarly, BP and UP nonresponders had significantly lower GM in OFC than their responder counterparts (Wilcoxon test, BP: responders, mean $(\mathrm{SD})=0.04(0.02)$, nonresponders mean $(\mathrm{SD})=-0.03(0.02), \chi^{2}=5.63, p=0.02 ;$ UP: responders, mean $(\mathrm{SD})=0.01(0.01)$, nonresponders mean $(\mathrm{SD})=-0.04$ $\left.(0.02), \chi^{2}=5.73, p=0.02\right)$. In addition, the differential relation between amygdala and OFC gluMI according to treatment response (responders and nonresponders) was maintained in both the UP and BP diagnostic subgroups (gluMI $\times$ group interactions, UP: $t=2.13, p=0.05 ; \mathrm{BP}$ : $t=2.40 ; p=0.05$ ).

In summary, the results suggest that alterations in brain metabolism and structure influence the response to TMS. Response to prefrontal TMS might depend on OFC volume and amygdala functioning. Further research is needed to determine the predictive value of such a functional pattern determined at an individual level in patients with resistant depression referred to TMS therapy.

\section{ACKNOWLEDGEMENTS}

This study was supported by Grant PHRC/AOM-98099 from the Assistance Publique-Hôpitaux de Paris (AP-HP) and the French Health Ministry; Grant INSERM-PROGRES A99013LS from the French Institute for Health and Medical Research (INSERM), and an AP-HP/INSERM interface grant (M-L Paillère-Martinot); Dr D Ringuenet was supported by the 'Fondation pour la Recherche Médicale' (FRM) and the Atomic Energy Commission (CEA). We are grateful to Vincent Brulon for image acquisition, Thierry Delzescaux and Claude Comtat for help with image processing, and Edouard Duchesnay for help with Brainvisa.

\section{DISCLOSURE}

The authors declare no conflict of interest. Over the past 3 years, Dr Paillère Martinot has received compensation from Bristol-Meyers-Squibb, Lilly, and Sanofi companies. Dr Artiges has received compensation from Janssen Laboratory; and Dr Galinowski from Ardix, Lilly, and Sanofi companies. 


\section{REFERENCES}

Annett M (1970). A classification of hand preference by association analysis. Br J Psychol 61: 303-321.

Ashburner J, Friston KJ (2005). Unified segmentation. Neuroimage 26: 839-851.

Barrett J, Della-Maggiore V, Chouinard PA, Paus T (2004). Mechanisms of action underlying the effect of repetitive transcranial magnetic stimulation on mood: behavioral and brain imaging studies. Neuropsychopharmacology 29: 1172-1189.

Brakemeier EL, Wilbertz G, Rodax S, Danker-Hopfe H, Zinka B, Zwanzger $\mathrm{P}$ et al (2008). Patterns of response to repetitive transcranial magnetic stimulation (rTMS) in major depression: replication study in drug-free patients. J Affect Disord 108: 59-70.

Brooks 3rd JO, Hoblyn JC, Woodard SA, Rosen AC, Ketter TA (2009). Corticolimbic metabolic dysregulation in euthymic older adults with bipolar disorder. J Psychiatr Res 43: 497-502.

Buchbaum MS, Buchbaum BR, Hazlett EA, Haznedar MM, Newmark R, Tang CY et al (2007). Relative glucose metabolic rate higher in white matter in patients with schizophrenia. Am J Psychiatry 164: 1072-1081.

Burgmer M, Pogatzki-Zahn E, Gaubitz M, Wessoleck E, Heuft G, Pfleiderer B (2009). Altered brain activity during pain processing in fibromyalgia. Neuroimage 44: 502-508.

Casanova R, Srikanth R, Baer A, Laurienti PJ, Burdette JH, Hayasaka $S$ et al (2007). Biological parametric mapping: a statistical toolbox for multimodality brain image analysis. Neuroimage 34: 137-143.

Chanraud S, Martelli C, Delain F, Kostogianni N, Douaud G, Aubin $\mathrm{HJ}$ et al (2007). Brain morphometry and cognitive performance in detoxified alcohol-dependents with preserved psychosocial functioning. Neuropsychopharmacology 32: 429-438.

Cohen J (1988). Statistical Power Analysis for the Behavioral Sciences. L Erlbaum Associates: Hillsdale, NJ.

Drevets WC (1999). Prefrontal cortical-amygdalar metabolism in major depression. Ann NY Acad Sci 877: 614-637.

Drevets WC (2000). Neuroimaging studies of mood disorders. Biol Psychiatry 48: 813-829.

Drevets WC, Bogers W, Raichle ME (2002). Functional anatomical correlates of antidepressant drug treatment assessed using PET measures of regional glucose metabolism. Eur Neuropsychopharmacol 12: 527-544.

Drevets WC, Price JL, Simpson JR, Todd RD, Reich T, Vannier M et al (1997). Subgenual prefrontal cortex abnormalities in mood disorders. Nature 386: 824-827.

Fregni F, Marcolin MA, Myczkowski M, Amiaz R, Hasey G, Rumi DO et al (2006). Predictors of antidepressant response in clinical trials of transcranial magnetic stimulation. Int J Neuropsychopharmacol 9: 641-654.

George MS, Wassermann EM, Williams WA, Callahan A, Ketter TA, Basser P et al (1995). Daily repetitive transcranial magnetic stimulation (rTMS) improves mood in depression. NeuroReport 6: $1853-1856$.

Greden JF (2001). The burden of disease for treatment-resistant depression. J Clin Psychiatry 62: 26-31.

Hamilton M (1960). A rating scale for depression. J Neurol Neurosurg Psychiatry 23: 56-62.

Herrmann LL, Ebmeier KP (2006). Factors modifying the efficacy of transcranial magnetic stimulation in the treatment of depression: a review. J Clin Psychiatry 67: 1870-1876.

Hooker CI, Knight RT (2006). The role of the lateral orbitofrontal cortex in the inhibitory control of emotion. In: Zald DH, Rauch SL (eds). The Orbitofrontal Cortex. Oxford University Press: Oxford, 307-324.

Hornig M, Mozley PD, Amsterdam JD (1997). HMPAO SPECT brain imaging in treatment-resistant depression. Prog Neuropsychopharmacol Biol Psychiatry 21: 1097-1114.
Houenou J, Wessa M, Douaud G, Leboyer M, Chanraud S, Perrin M et al (2007). Increased white matter connectivity in euthymic bipolar patients: diffusion tensor tractography between the subgenual cingulate and the amygdalo-hippocampal complex. Mol Psychiatry 12: 1001-1010.

Ketter TA, Kimbrell TA, George MS, Dunn RT, Speer AM, Benson BE et al (2001). Effects of mood and subtype on cerebral glucose metabolism in treatment-resistant bipolar disorder. Biol Psychiatry 49: 97-109.

Konarski JZ, Kennedy SH, McIntyre RS, Rafi-Tari S, Soczynska JK, Mayberg HS (2007). Relationship between regional brain metabolism, illness severity and age in depressed subjects. Psychiatry Res 155: 203-210.

Lam RW, Chan P, Wilkins-Ho M, Yatham LN (2008). Repetitive transcranial magnetic stimulation for treatment-resistant depression: a systematic review and metaanalysis. Can J Psychiatry 53: 621-631.

Langguth B, Wiegand R, Kharraz A, Landgrebe M, Marienhagen J, Frick U et al (2007). Pre-treatment anterior cingulate activity as a predictor of antidepressant response to repetitive transcranial magnetic stimulation (rTMS). Neuro Endocrinol Lett 28: 633-638.

Lisanby SH, Husain MM, Rosenquist PB, Maixner D, Gutierrez R, Krystal A et al (2009). Daily left prefrontal repetitive transcranial magnetic stimulation in the acute treatment of major depression: clinical predictors of outcome in a multisite, randomized controlled clinical trial. Neuropsychopharmacology 34: 522-534.

Little JT, Ketter TA, Kimbrell TA, Dunn RT, Benson BE, Willis MW et al (2005). Bupropion and venlafaxine responders differ in pretreatment regional cerebral metabolism in unipolar depression. Biol Psychiatry 57: 220-228.

Loo CK, Mitchell PB (2005). A review of the efficacy of transcranial magnetic stimulation (TMS) treatment for depression, and current and future strategies to optimize efficacy. J Affect Disord 88: 255-267.

Maldjian JA, Laurienti PJ, Kraft RA, Burdette JH (2003). An automated method for neuroanatomic and cytoarchitectonic atlas-based interrogation of fMRI data sets. Neuroimage 19: 1233-1239.

Martinot JL (1992). Drug washout issues in studies of cerebral metabolism by positron emission tomography in psychiatric patients. J Neural Transm (Suppl) 37: 27-38.

Mayberg HS (1997a). Limbic-cortical dysregulation: a proposed model of depression. J Neuropsychiatry Clin Neurosci 9: 471-481.

Mayberg HS, Brannan SK, Mahurin RK, Jerabek PA, Brickman JS, McGinnis S et al (1997b). Cingulate function in depression: a potential predictor of treatment response. NeuroReport 8: 1057-1061.

Mayberg HS, Lewis PJ, Regenold W, Wagner Jr HN (1994). Paralimbic hypoperfusion in unipolar depression. J Nucl Med 35: 929-934.

Montgomery SA, Asberg M (1979). A new depression scale designed to be sensitive to change. Br J Psychiatry 134: 382-389.

Mottaghy FM, Keller CE, Gangitano M, Ly J, Thall M, Parker JA et al (2002). Correlation of cerebral blood flow and treatment effects of repetitive transcranial magnetic stimulation in depressed patients. Psychiatry Res 115: 1-14.

Nadeau SE, McCoy KJ, Crucian GP, Greer RA, Rossi F, Bowers D et al (2002). Cerebral blood flow changes in depressed patients after treatment with repetitive transcranial magnetic stimulation: evidence of individual variability. Neuropsychiatry Neuropsychol Behav Neurol 15: 159-175.

O’Reardon JP, Solvason HB, Janicak PG, Sampson S, Isenberg KE, Nahas Z et al (2007). Efficacy and safety of transcranial magnetic stimulation in the acute treatment of major depression: a multisite randomized controlled trial. Biol Psychiatry 62: 1208-1216. 
Paillère Martinot ML, Galinowski A, Ringuenet D, Gallarda T, Lefaucheur JP, Bellivier F et al (2009). Influence of prefrontal target region on the efficacy of repetitive transcranial magnetic stimulation in patients with medication-resistant depression: a $\left[{ }^{18} \mathrm{~F}\right]$-fluorodeoxyglucose PET and MRI study. Int J Neuropsychopharmacol 9: 1-15.

Pascual-Leone A, Rubio B, Pallardó F, Catalá MD (1996). Rapidrate transcranial magnetic stimulation of left dorsolateral prefrontal cortex in drug-resistant depression. Lancet 347: 233-237.

Savitz J, Drevets WC (2009). Bipolar and major depressive disorder: neuroimaging the developmental-degenerative divide. Neurosci Biobehav Rev 33: 699-771.

Saxena S, Brody AL, Ho ML, Alborzian S, Maidment KM, Zohrabi N et al (2002). Differential cerebral metabolic changes with paroxetine treatment of obsessive-compulsive disorder vs major depression. Arch Gen Psychiatry 59: 250-261.

Schmahmann JD, Pandya DN (2006). Uncinate fasciculus. In: Schmahmann JD, Pandya DN (eds). Fiber Pathways of the Brain. Oxford University Press: New York, 419-425.

Schultz W, Tremblay L, Hollerman JR (1998). Reward prediction in primate basal ganglia and frontal cortex. Neuropharmacology 37: 421-429.

Seminowicz DA, Mayberg HS, McIntosh AR, Goldapple K, Kennedy S, Segal Z et al (2004). Limbic-frontal circuitry in major depression: a path modeling metanalysis. Neuroimage 22: 409-418.

Sheehan DV, Lecrubier Y, Sheehan KH, Amorim P, Janavs J, Weiller E et al (1998). The Mini-International Neuropsychiatric Interview (M.I.N.I.): the development and validation of a structured diagnostic psychiatric interview for DSM-IV and ICD-10. J Clin Psychiatry 59(Suppl 20): 22-33; quiz 34-57.

Talairach J, Tournoux P (1988). Co-Planar Stereotaxic Atlas of the Human Brain: An Approach to Medical Cerebral Imaging. Thieme Medical Publishers: New York.

Teneback CC, Nahas Z, Speer AM, Molloy M, Stallings LE, Spicer KM et al (1999). Changes in prefrontal cortex and paralimbic activity in depression following two weeks of daily left prefrontal TMS. J Neuropsychiatry Clin Neurosci 11: 426-435.

Thase ME, Rush AJ (1997). When at first you don't succeed: sequential strategies for antidepressant nonresponders. J. Clin. Psychiatry 58(Suppl 13): 23-29.

Tyrer P, Owen RT, Cicchetti DV (1984). The brief scale for anxiety: a subdivision of the comprehensive psychopathological rating scale. J Neurol Neurosurg Psychiatry 47: 970-975.

Videbech P (2000). PET measurements of brain glucose metabolism and blood flow in major depressive disorder: a critical review. Acta Psychiatr Scand 101: 11-20.

Zald DH (2003). The human amygdala and the emotional evaluation of sensory stimuli. Brain Res Brain Res Rev 41: 88-123.

Supplementary Information accompanies the paper on the Neuropsychopharmacology website (http://www.nature.com/npp) 